\title{
The effect of AGN feedback on the X-ray morphologies of clusters: Simulations vs. observations
}

\author{
Gayoung Chon ${ }^{1}$, Ewald Puchwein ${ }^{2}$, and Hans Böhringer ${ }^{1}$ \\ 1 Max-Planck-Institut für extraterrestrische Physik, 85748 Garching, Germany \\ e-mail: gchon@mpe.mpg.de \\ 2 Institute of Astronomy and Kavli Institute for Cosmology, University of Cambridge, Madingley Road, Cambridge CB3 0HA, UK \\ Received 16 March 2016 / Accepted 19 May 2016
}

\begin{abstract}
Clusters of galaxies probe the large-scale distribution of matter and are a useful tool to test the cosmological models by constraining cosmic structure growth and the expansion of the Universe. It is the scaling relations between mass observables and the true mass of a cluster through which we obtain the cosmological constraints by comparing to theoretical cluster mass functions. These scaling relations are, however, heavily influenced by cluster morphology. The presence of the slight tension in recent cosmological constraints on $\Omega_{\mathrm{m}}$ and $\sigma_{8}$ based on the CMB and clusters has boosted the interests in looking for possible sources for the discrepancy. Therefore we study here the effect of active galactic nucleus (AGN) feedback as one of the major mechanisms modifying the cluster morphology influencing scaling relations. It is known that AGN feedback injects energies up to $10^{62} \mathrm{erg}$ into the intracluster medium, controls the heating and cooling of a cluster, and re-distributes cold gas from the centre to outer radii. We have also learned that cluster simulations with AGN feedback can reproduce observed cluster properties, for example, the X-ray luminosity, temperature, and cooling rate at the centre better than without the AGN feedback. In this paper using cosmological hydrodynamical simulations we investigate how the AGN feedback changes the X-ray morphology of the simulated systems, and compare this to the observed Representative $X M M-N e w t o n$ Cluster Structure Survey (REXCESS) clusters. We apply two substructure measures, centre shifts $(w)$ and power ratios (e.g. $P_{3} / P_{0}$ ), to characterise the cluster morphology, and find that our simulated clusters are more substructured than the observed clusters based on the values of $w$ and $P_{3} / P_{0}$. We also show that the degree of this discrepancy is affected by the inclusion of AGN feedback. While the clusters simulated with the AGN feedback are in much better agreement with the REXCESS $L_{X}-T$ relation, they are also more substructured, which increases the tension with observations. When classified as non-relaxed or relaxed according to their $w$ and $P_{3} / P_{0}$ values, we find that there are no relaxed clusters in the simulations with the AGN feedback. This suggests that not only global cluster properties, like $L_{\mathrm{X}}$ and $\mathrm{T}$, and radial profiles should be used to compare and to calibrate simulations with observations, but also substructure measures like centre shifts and power ratios. Finally, we discuss what changes in the simulations might ease the tension with observational constraints on these quantities.
\end{abstract}

Key words. galaxies: clusters: general - galaxies: clusters: intracluster medium - X-rays: galaxies: clusters - methods: numerical cosmology: observations

\section{Introduction}

Clusters of galaxies have played an important role in accessing the distribution of dark matter on scales from $\mathrm{Mpc}$ up to hundred Mpc, for example Collins et al. (2000), Chon et al. (2013), Böhringer et al. (2015), and have also been proven to be effective tracers of cosmological evolution through the cluster mass function that allows testing of cosmological models (see e.g. Vikhlinin et al. 2009; Kravtsov \& Borgani 2012; Böhringer et al. 2014). The success of cosmological studies with clusters of galaxies heavily relies on the fact that mass observables are a clean probe of the total mass of a system. As shown explicitly by Böhringer et al. (2014) the scaling relation that connects the measured X-ray luminosity to the total mass has a large influence on the cosmological constraints, most sensitively through $\sigma_{8}$ and $\Omega_{\mathrm{m}}$. Hence it is crucial to understand scaling relations and their statistical properties as accurately as possible.

In our previous study we showed that the dynamical states or equivalently morphologies of clusters help us to understand the scatter introduced in scaling relations (Chon et al. 2012). For instance we showed that the normalisation of scaling relations is systematically higher or lower depending on the dynamical state of a cluster. This is very interesting in light of an apparent tension of the constraints on $\sigma_{8}$ and $\Omega_{\mathrm{m}}$ from the analysis of the comic microwave background (CMB) and cluster counts (Planck Collaboration XXIV 2016; Planck Collaboration XIII 2016; Hasselfield et al. 2013; Vikhlinin et al. 2009; Böhringer et al. 2014) because it is this normalisation parameter of the scaling relation that may be able to ease the tension in addition to the much-discussed hydrostatic mass bias.

One of the major mechanisms that modifies the distribution of the intracluster medium (ICM) is active galactic nucleus (AGN) feedback. The lack of highly cooling gas at the centres of clusters in X-ray observations provides a direct evidence that there is a heating mechanism that prevents the ICM from over-cooling (Peterson et al. 2001; Böhringer et al. 2002). The most viable source for this heating is provided by central black holes injecting energy into the ICM. There are two known modes of AGN feedback, radio and quasar modes, depending on the accretion rates of central black holes (see e.g. the reviews by McNamara \& Nulsen 2007, 2012). The quasar mode is effective in the early phase of the AGN evolution, when the black hole mass accretion rate is high and most of the feedback energy is emitted as radiation. For clusters of galaxies the most relevant mechanism is the radio mode in operation at low black 
hole mass accretion rates. In this mode the feedback energy is released primarily as mechanical power that heats the atmosphere through buoyant bubbles rising from the centre initiated by energetic AGN jet events. Also the heating by shock waves triggered by the AGN jets has been observed in a few cases.

Springel et al. (2005a) developed techniques to incorporate the AGN feedback in cosmological hydrodynamics simulations and modifications were made, for example, by Sijacki \& Springel (2006), Sijacki et al. (2007), and Fabjan et al. (2010), which included both the quasar and radio feedback modes. There are several studies that investigated the effect of AGN feedback based on the X-ray properties of clusters and groups including scaling relation, temperature, and metal abundance profiles (see e.g. Puchwein et al. 2008; Fabjan et al. 2010; McCarthy et al. 2010; Planelles et al. 2014). However, none of these studies directly investigated the effect of AGN feedback on the morphologies of clusters and compared them to observations; this is the main aim of this paper.

In a previous study, Puchwein et al. (2008) showed that including AGN feedback in the simulations of the formation of galaxy clusters reproduces some of the important scaling relations of cluster parameters from X-ray observations, which are not reproduced without the effect of AGN feedback. In particular the relation of the X-ray luminosity and the intracluster plasma temperature as well as the intracluster gas mass fraction were well matched to observations for the simulations including AGN feedback. Here we take this simulation result and explore the effect of the AGN feedback on the morphology of X-ray clusters.

We employ two common substructure measures, the power ratios and centre shifts, which are well tested for the X-ray observations and simulations (see e.g. Böhringer et al. 2010; Chon et al. 2012; Mahdavi et al. 2013; Rasia et al. 2013) to determine the degree of substructure quantitatively and compare the results from simulations with and without AGN feedback to $\mathrm{X}$-ray observations. For the X-ray observations we consider the REXCESS clusters. They form a statistical sample that is closer to a volume-limited sample drawn from the ROSAT-ESO Flux Limited X-ray galaxy cluster Survey (REFLEX; Böhringer et al. 2001, 2013; Chon \& Böhringer 2012), all of which have deep exposures with XMM-Newton observations. The REXCESS clusters have been scrutinised for X-ray scaling relations and morphologies (Pratt et al. 2009; Böhringer et al. 2010), hence provide a good observational basis to compare to simulations.

The paper is structured as follows. In Sect. 2 we describe the simulation data and the observational data, and provide a brief summary of the substructure measures in Sect. 3. In Sect. 4 we study the AGN feedback effect on the cluster morphology in the simulations and compare these results to the REXCESS. We conclude the paper with a summary in Sect. 5 .

\section{Sample description}

\subsection{The simulated cluster sample}

We use the galaxy cluster and group sample from Puchwein et al. (2008), which consists of 21 re-simulations of Millennium simulation (Springel et al. 2005b) halos performed with the zoomin technique. The same flat $\Lambda$ cold dark matter cosmology as in the parent Millennium simulation was adopted, $\Omega_{\mathrm{m}}=0.25$, $n_{s}=1, \sigma_{8}=0.9$, and $\Omega_{b}=0.04136$. The resolution of the resimulated halos with virial masses below $2 \times 10^{14} h^{-1} M_{\odot}$ is as follows: the DM particle mass is $m_{\mathrm{DM}}=3.1 \times 10^{7} h^{-1} M_{\odot}$, the gas particle mass is $m_{\text {gas }}=6.2 \times 10^{6} h^{-1} M_{\odot}$ and the physical softening is $\epsilon=2.5 h^{-1} \mathrm{kpc}$. A lower resolution was used with a DM particle mass of $m_{\mathrm{DM}}=1.1 \times 10^{8} h^{-1} M_{\odot}$ and a gas particle mass of $m_{\mathrm{gas}}=2.1 \times 10^{7} h^{-1} M_{\odot}$ for the four most massive clusters. All re-simulations include hydrodynamics, radiative cooling assuming a primordial gas composition, heating by an external UV background, star formation, and supernovae feedback. The selection of this sample was based only on halo mass covering a large range from $M_{200}=8 \times 10^{12} M_{\odot}$ to $1.5 \times 10^{15} M_{\odot}$. Here and throughout this work the densities quoted in the subscript indices of spherical overdensity masses and radii are given in units of the critical density of the Universe at the cluster redshift. The simulations employ a so-called traditional entropy-conserving formulation of smoothed particle hydrodynamics (SPH; Springel \& Hernquist 2002). No artificial conduction or mixing prescription is used in this scheme. The runs were carried out with the P-Gadget 3 code (last described in Springel et al. 2005b). For each halo two kinds of re-simulations are available. They were performed either with or without a model for the growth of supermassive black holes (BHs) and associated feedback processes as in Sijacki et al. (2007; also see Springel et al. 2005a). The stellar components of these clusters were studied in Puchwein et al. (2010), while their lensing properties were investigated in Mead et al. (2010).

To incorporate $\mathrm{BH}$ growth and feedback it was assumed that any halo above a mass of $5 \times 10^{10} h^{-1} M_{\odot}$ contains a seed $\mathrm{BH}$ with a mass of $10^{5} h^{-1} M_{\odot}$. The BHs are allowed to grow by mergers with other BHs and by accretion of gas. The prescription of the latter is motivated by the Bondi-Hoyle-Lyttleton model, but with the Eddington limit additionally imposed.

Two modes of AGN feedback were adopted depending on the $\mathrm{BH}$ accretion rate: above 0.01 of the Eddington rate the quasar mode and below the radio mode. In the quasar mode where feedback is assumed to be predominantly radiative, a low coupling efficiency of $0.5 \%$ of the rest mass energy of the accreted gas is used. This energy is continuously injected in the form of thermal energy into nearby gas particles. In the radio mode, feedback is assumed to happen by recurrently inflating AGN-heated bubbles into the ICM. We employ a larger mechanical coupling efficiency of $2 \%$ of the accreted rest mass energy, which is in good agreement with X-ray observations of elliptical galaxies (Allen et al. 2006). The radio mode feedback energy is injected, i.e. a bubble event is triggered, whenever the mass of the $\mathrm{BH}$ has grown by a factor $\Delta M_{\mathrm{BH}} / M_{\mathrm{BH}} \geq$ $10^{-4}$. The accumulated feedback energy is then injected thermally into a spherical bubble with a radius given by $R_{\text {bub }}=$ $R_{\text {bub }, 0}\left[\left(E_{\text {bub }} / E_{\text {bub }, 0}\right) /\left(\rho_{\text {bub }} / \rho_{\text {bub }, 0}\right)\right]^{1 / 5}$, where the default bubble radius $R_{\text {bub }, 0}=30 h^{-1} \mathrm{kpc}$ at a bubble energy of $E_{\text {bub, } 0}=10^{55} \mathrm{erg}$ and an ICM density of $\rho_{\text {bub, } 0}=10^{13} h^{-2} M_{\odot} \mathrm{Mpc}^{-3}$ fixes the overall normalisation of the radii of bubbles. The scaling with the bubble energy, $E_{\text {bub }}$, and the ICM density at the bubble position, $\rho_{\text {bub }}$, is motivated by solutions of the expansion of radio cocoons (e.g. Heinz et al. 1998) and ensures that more energetic feedback events result in larger bubbles, while a denser ICM confines the bubble size more strongly. The centre of the bubble is chosen randomly within a radius of $5 / 3 \times R_{\text {bub }}$ around the $\mathrm{BH}$. The adopted $\mathrm{BH}$ model leads to a self-regulated $\mathrm{BH}$ growth as shown in Sijacki et al. (2007).

\subsection{X-ray properties of simulated and observed clusters}

Two sets of 21 clusters, with and without AGN feedback, were used to create X-ray surface brightness images in three projections resulting in 126 clusters images. The cluster mass range described above corresponds to X-ray spectroscopic temperatures between 0.4 to $7.4 \mathrm{keV}$. A constant metallicity of 
$0.3 Z_{\odot}$ was assumed in the synthetic $\mathrm{X}$-ray analysis. The $\mathrm{X}$-ray images were produced in the $[0.5-2.0] \mathrm{keV}$ band.

For the comparison with observations we used the representative XMM-Newton cluster structure survey (REXCESS) data (Böhringer et al. 2007) comprised of 31 galaxy clusters that are selected to be morphologically representative (Böhringer et al. 2010). The cluster selection is closer to volume limited than flux limited, which is important to note, as these selections do affect the distributions of the morphologies of clusters (Chon et al., in prep.). The lower limit of the temperature in this sample is $2 \mathrm{keV}$.

While our results are shown for all of the simulated clusters, we make a closer comparison to the observations with the four simulated clusters that fall into the same temperature or mass range as the REXCESS sample.

\section{Structural analysis}

We concentrate on two methods to characterise the degree of substructure: the power ratios (Buote \& Tsai 1995) and centre shifts (e.g. Mohr et al. 1993; Poole et al. 2006). We use the same technique as in Böhringer et al. (2010) with modifications as descried in Chon et al. (2012) such that the final classification of the morphology reflects the mean dynamics over the entire cluster out to $r_{500}$.

\subsection{Power ratio calculation}

The power ratio method first introduced by Buote \& Tsai (1995) was motivated by the assumption that the X-ray surface brightness closely traces the projected two-dimensional mass distribution of a cluster. A multipole decomposition of such a projected mass distribution provides moments that are identified as power ratios after normalisation by the zeroth moment. In practice the power ratio analysis is applied to the surface brightness distribution.

The moments $P_{m}$ are defined as

$$
\begin{aligned}
& P_{0}=\left[a_{0} \ln \left(R_{\mathrm{ap}}\right)\right]^{2} \\
& P_{m}=\frac{1}{2 m^{2} R_{\mathrm{ap}}^{2 m}}\left(a_{m}^{2}+b_{m}^{2}\right),
\end{aligned}
$$

where $R_{\text {ap }}$ is the aperture radius in units of $r_{500}$. The moments $a_{m}$ and $b_{m}$ are calculated by

$a_{m}(r)=\int_{r \leq R_{\mathrm{ap}}} \mathrm{d} \boldsymbol{x} S(\boldsymbol{x}) r^{m} \cos (m \phi)$

and

$$
b_{m}(r)=\int_{r \leq R_{\mathrm{ap}}} \mathrm{d} \boldsymbol{x} S(\boldsymbol{x}) r^{m} \sin (m \phi),
$$

where $S(\boldsymbol{x})$ is the X-ray surface brightness image, and the integral extends over all pixels inside the aperture radius. Thus, $a_{0}$ in Eq. (1) is the total radiation intensity inside the aperture radius.

Since all $P_{m}$ are proportional to the total intensity of the X-ray image, all moments are normalised by $P_{0}$ resulting in the so-called power ratios, $P_{m} / P_{0}$. For brevity we refer to $P_{m} / P_{0}$ as $P_{m}$ in the rest of the paper.

Similar to all previous studies, we only make use of the lowest moments from $P_{2}$ to $P_{4}$. Before the multipole moments are determined, the centre for the calculations is found by determining the centre of mass in the vignetting and gap corrected surface brightness images. The dipole, $P_{1}$, which is checked during the calculations, should therefore vanish. $P_{2}$ describes the quadrupole of the surface brightness distribution, which is not necessarily a measure of substructure. In practice, low-tomoderate values of $P_{2}$ are found for regular elliptical clusters, while higher values of $P_{2}$ are a sign of cluster mergers. The lowest power ratio moment providing a clear substructure measure is thus $P_{3} . P_{4}$ describes substructure on slightly finer scales and is found to be correlated with $P_{2}$ here and in previous studies (Chon et al. 2012).

Typically $P_{m}(r)$ is evaluated at $r_{500}$. Because of the $r^{m}$ weighting in Eqs. (3) and (4), it is then predominantly influenced by the dynamical state in the outskirts of a cluster. To overcome this strong bias to the outer part of the cluster, we measure the averaged power ratios at ten radii defined by fractions of $r_{500}$. Further detailed discussions and comparisons to other definitions are found in Chon et al. (2012).

In the case of observed clusters the uncertainty of the power ratio measurement and the influence of photon noise are studied by simulations in which an additional Poisson noise is imposed on the count images with background. This "Poissonisation" is equivalent to the Poisson noise introduced by the observation involving a finite number of detected photons. We interpret the variance of the power ratio results from the simulations as the measurement uncertainty. We then make the assumption that the additional power introduced by the Poissonisation is similar to the extra power in the power ratios introduced by the photon shot noise of the observation. We therefore subtract the additional noise found in the mean of all simulations compared to the observations from the observational result.

\subsection{Centre shifts}

The centre shift measures the stability of the X-ray centre calculated at different radii and is formulated as (Poole et al. 2006)

$w=\left[\frac{1}{N-1} \sum\left(\Delta_{i}-\langle\Delta\rangle\right)^{2}\right]^{1 / 2} \times \frac{1}{r_{500}}$,

where $\Delta_{i}$ is the distance between the mean centroid and the centroid of the $i$ th aperture.

The centroid of each aperture is found by determining the centre of mass of the photon distribution in each aperture, which was already used for the centring prior to the power ratio determination. The resulting $w$ is then the standard deviation of the different centre shifts (in units of $r_{500}$ ). We use the mean centroid value of all apertures as the reference centre.

For the X-ray observations of clusters the uncertainties in the centre shifts and in the $w$ parameter are determined with the same simulations as the uncertainties of the power ratios, i.e., by using Poissonised re-sampled cluster X-ray images. The standard deviation of the $w$ parameter in the simulation is used as an estimate of the measurement uncertainties. We do not use the noise-bias subtracted $w$-parameter as in the case of the power ratios since the bias correction is mostly much smaller than the errors and the bias correction does not shift the $w$-parameter to alter the classification of the cluster morphology.

We performed end-to-end Monte Carlo simulations of the Poissonised data analysed exactly the same way as we calculate the power ratios and centre shifts with the original X-ray data. This ensures that, for example, the systematics introduced by the photon shot noise is properly taken into account in the parameter uncertainties. Those simulated clusters in the same mass or equivalent temperature range as those of the REXCESS 


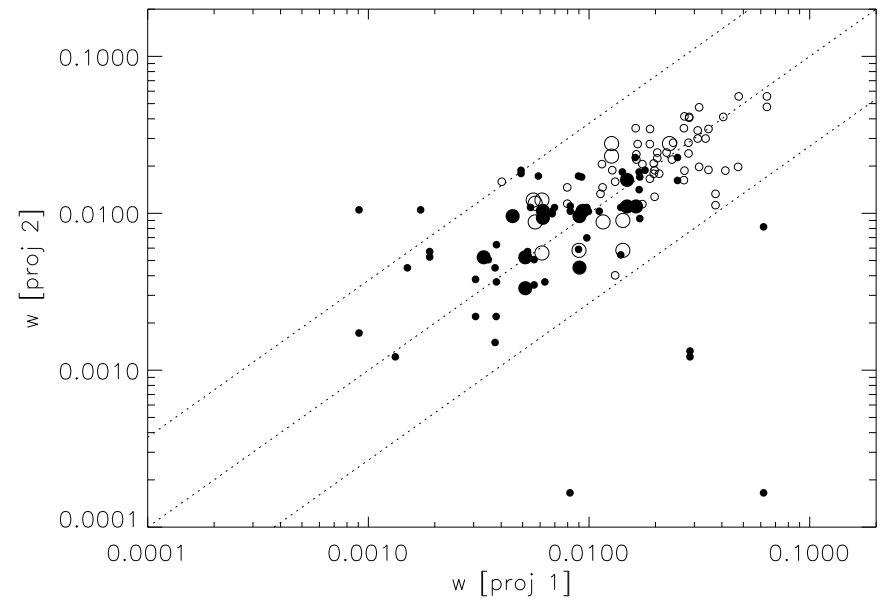

Fig. 1. Comparison of the results of the centre shift analysis for two projections of the same cluster. Filled symbols show the clusters without AGN feedback and open symbols those with the feedback. Larger circles represent those clusters above $2 \mathrm{keV}$ as in the REXCESS sample. The central dotted line indicates a one-to-one relation, enclosed by three sigma scatter.

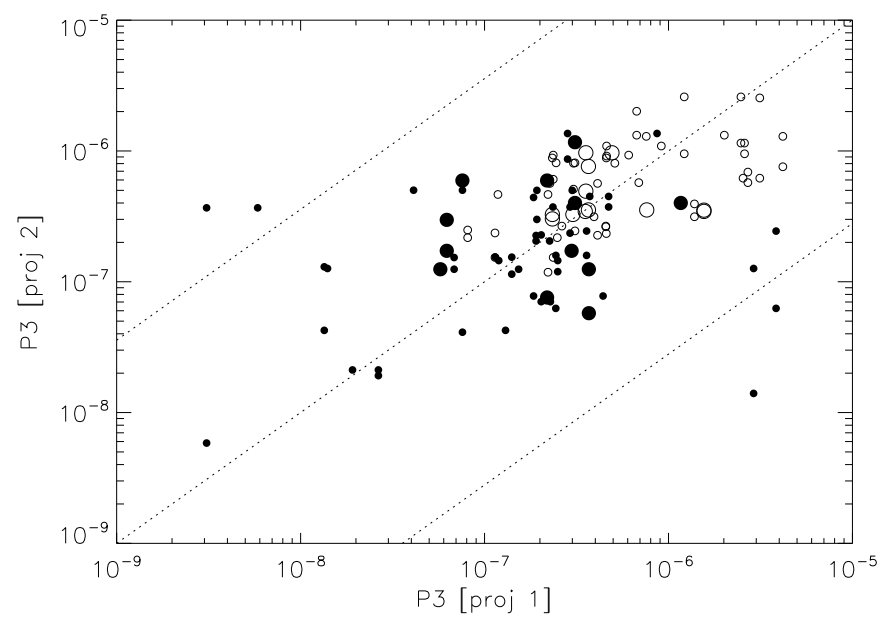

Fig. 2. Comparison of the power ratio, P3, for two projections of the same cluster. Symbols are defined as those in Fig. 1.

data are represented by larger symbols in the figures where this distinction is necessary.

\subsection{Variations of observed substructure due to projections}

The line-of-sight projection of a cluster leaves the properties related to the third dimension unconstrained. This ambiguity introduces scatter in the morphology parameters for an individual cluster. The degree of scatter can be studied with the substructure measures from three different projections of the same cluster.

We show the results for the power ratio, $P_{3}$, and for the centre shift parameter in Figs. 1 and 2 for all 126 cases. Both figures show a log-log correlation between different projections with the three sigma scatter drawn as dotted lines around a central dotted line denoting an one-to-one relation. The clusters with AGN feedback (open circles) have a higher value of $w$ and a smaller scatter than those without (filled). The scatter is smaller for more massive (large circles) than for the less massive clusters at least in the runs without AGN. There are nine clusters whose $w$ value in one projection is significantly higher than in the other one, which are located outside the three sigma lines. It is interesting
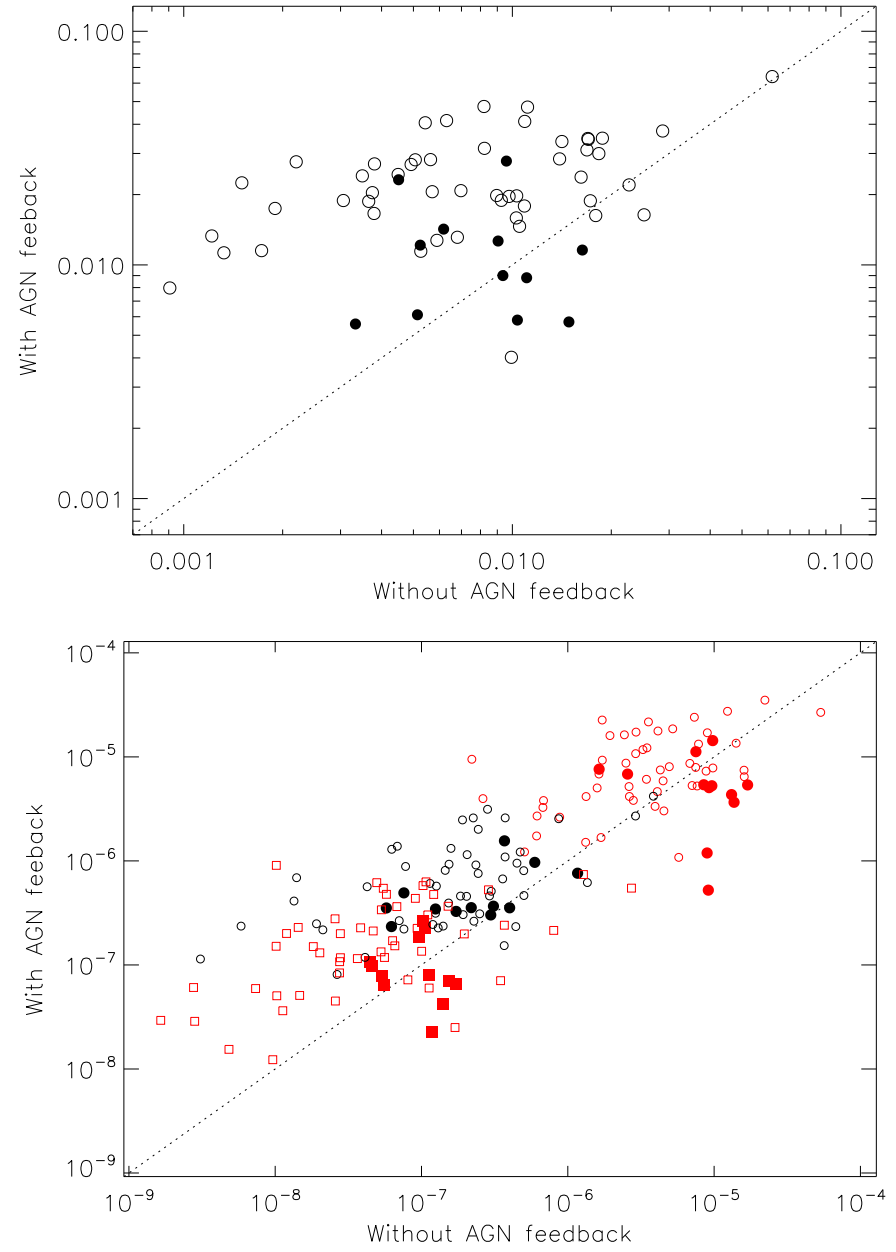

Fig. 3. Top: comparison of the centre shift values. Bottom: comparison of the power ratios, $P_{2}$ (red circles), $P_{3}$ (black circles) and $P_{4}$ (red squares). In both panels massive clusters (filled) and low mass clusters (open circles) are shown together with an equality line (dashed).

to note that they are on the least massive end of the mass range in the simulations. Eight of those are less massive clusters without AGN feedback and one is with AGN feedback. On average the scatter of $w$ is smaller than that of $P_{3}$, which may indicate that $w$ has a stronger constraining power of substructure than $P_{3}$, at least for a moderately sized sample, despite those five clusters with very large departures from the one-to-one relation in $w$.

The influence of AGN feedback is investigated in more detail in the next section.

\section{The effect of AGN feedback}

\subsection{Simulation}

Figures 1 and 2 indicate that AGN feedback creates more substructure in the X-ray surface brightness maps, and we study here the degree of how much more substructure is introduced by the AGN feedback. Figure 3 compares directly the values of $w$ (top panel) and $P_{2}, P_{3}$ and $P_{4}$ (bottom) for simulations with and without AGN feedback for the same cluster in the same projection.

The upper panel clearly shows that low mass objects (open circles) typically have a higher $w$ value in runs with AGN feedback than in runs without. For massive clusters (filled circles) this offset becomes less significant. This finding is not too surprising as the energy input of the AGN pushes the gas out of 
Table 1. Ratios of the substructure parameters between the runs with and without AGN feedback divided into two mass groups.

\begin{tabular}{ccccc}
\hline \hline & $w$ & $P_{2}$ & $P_{3}$ & $P_{4}$ \\
\hline Light & 10.66 & 3.80 & 7.69 & 7.01 \\
Massive & 1.69 & 1.09 & 2.69 & 1.32 \\
\hline
\end{tabular}

Notes. This division is done at $2 \mathrm{keV}$, which is the lower temperature limit of the REXCESS cluster sample. This roughly corresponds to a mass cut of $8 \times 10^{13} M_{\odot} / \mathrm{h}$.

the very central region and this has a larger effect on the global scale in low mass groups with shallower gravitational potentials compared to massive clusters where the deeper gravitational potential can confine the feedback effect more to the central region.

In Table 1 we list the mean ratios of the centre shifts and power ratios between the runs with and without AGN feedback. We find that the mean ratio of the $w$ values between with and without the AGN feedback is approximately six times larger in less massive clusters than that in massive clusters. More concretely the mean value of $w$ with the AGN feedback is 0.012 for the massive clusters and 0.025 for the less massive clusters. This suggests that the current implementation of the AGN feedback not only pushes the gas out to larger radii in less massive clusters but also produces a more asymmetric gas distribution. This larger effect in low mass objects is consistent with previous simulation results, and was found to be required to match observed cluster scaling relations and gas fractions at the low mass end (Puchwein et al. 2008).

We also show a similar trend for all three power ratios in the bottom panel of Fig. 3. The bulk mean amplitude of power ratios increases from $P_{4}$ to $P_{2}$. Overall the power ratios that are more heavily influenced by the inclusion of the AGN feedback are $P_{3}$ and $P_{4}$. For $P_{2}$ the less massive clusters have a mean ratio of 3.8 between the runs with and without AGN feedback while that of the massive clusters is 1.1 as shown in Table 1 . However, the effect of the AGN feedback is more pronounced in $P_{3}$ and $P_{4}$ as they measure the distortion of gas distribution on finer scales. As seen in the centre shifts we also find that the ratio is lower for massive clusters than the less massive ones, typically by a factor of three to five. The mean value of $P_{3}$ with AGN feedback is $5.34 \times 10^{-7}$ for the massive clusters and $9.10 \times 10^{-7}$ for the less massive clusters.

One primarily expects that AGNs heat the intracluster medium to prevent a massive cooling and condensation of gas in the centre, to disturb the distribution of the central gas, and to puff the cold gas out to a larger distance. Hence it is not surprising that clusters with AGN feedback show a larger degree of substructure in the gas distribution than those without and that the gas distribution of less massive systems are more affected by AGN feedback than that of massive clusters. We compare our findings in the simulations with the observations in the next section.

\subsection{Comparison to observations}

We use the REXCESS clusters (Böhringer et al. 2007) to compare the simulations to observations. In Fig. 4 we compare the $w$ and $P_{3}$ values for the simulations (black circles) and observations (red squares with errors). Overall there is a correlation between the two parameters with some scatter. A large number of the simulated clusters are located in the upper right corner of the $w-P_{3}$ plane suggesting more substructures. Among the

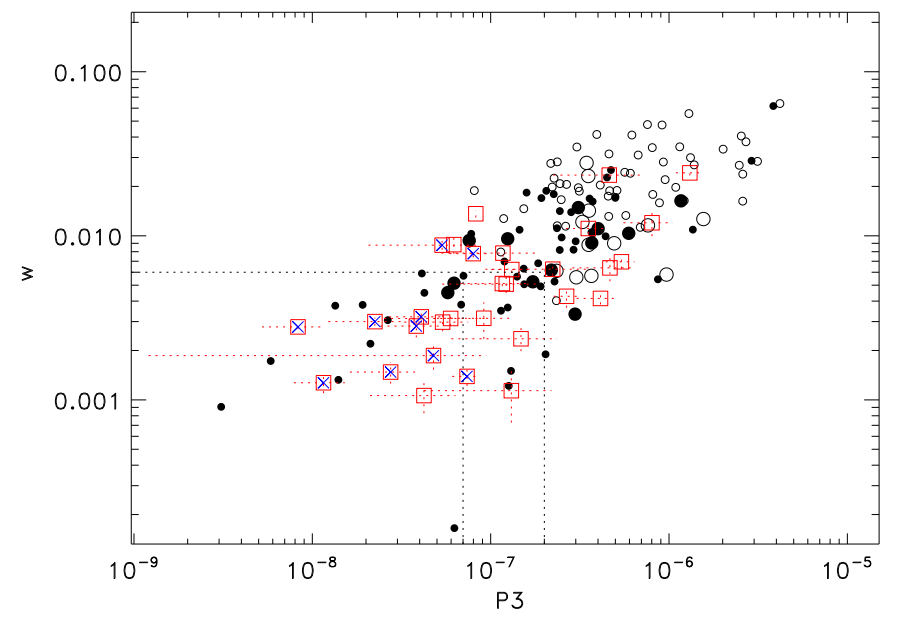

Fig. 4. Comparison of the centre shift parameter $w$ and power ratio $P_{3}$ for observations (red squares) and simulations (circles). Open circles indicate clusters with and filled circles without AGN feedback. The massive clusters are marked with larger circles. Among the observations cool-core clusters are indicated with blue crosses. Dotted lines give guidelines to distinguish different morphological types (see text for details).

simulated sample the clusters with the AGN feedback (open circles) are more substructured than those without (filled). Also the less massive clusters and groups (small circles) tend to be at the lower left or upper right corner of the figure displaying a larger range of cluster morphologies than for the massive clusters (large circles), which are distributed in the middle of the plot. Those REXCESS clusters identified as cool-core clusters (Pratt et al. 2009) are shown with the extra blue crosses. A fair comparison of the observations and simulations needs to be restricted to the same mass range (indicated by large circles for the simulations). This comparison would then bring the observations and simulations closer in the $w-P_{3}$ plane albeit retaining the tendency that the simulated clusters have still larger $w$ and $P_{3}$ parameters. The ratio of the mean $w$ parameters between the simulations without AGN feedback and the observations is 1.4 and that of $P_{3}$ is 1.6. These ratios increase to 1.9 for $w$ and 2.6 for $P_{3}$ if we consider the runs with AGN feedback instead.

The three dotted lines in Fig. 4 are defined and used in Chon et al. (2012) to classify the morphology of a cluster in a more qualitative way, which also proved useful for other purposes. This classification divides clusters into three groups, the disturbed $\left(w>6 \times 10^{-3}\right.$ or $\left.P_{3}>2 \times 10^{-7}\right)$, relaxed $\left(w<6 \times 10^{-3}\right.$ and $P_{3}<6 \times 10^{-8}$ ), and intermediate, which occupy the bottom narrow strip in Fig. 4. The REXCESS sample then contains 15 disturbed, 6 intermediate, and 10 relaxed clusters. As was found in Chon et al. (2012) this division of morphologies divides the sample into two similarly sized groups: disturbed and less disturbed. In contrast, $75 \%$ of the massive clusters simulated without AGN and all of the massive clusters simulated with AGN are disturbed. Hence we find a significant discrepancy in morphologies between the simulated and observed clusters, which gets larger when AGN feedback is included. This indicates that some relevant physics might be missing or is incorrectly treated in the simulations. Furthermore, the increased discrepancy in morphology in runs with AGN feedback might suggest that the way AGN feedback was introduced in the simulations was perhaps more violent than in nature and in particular introduced more asymmetric disturbances. If the energy deposition preserved more of the symmetry, the morphology 


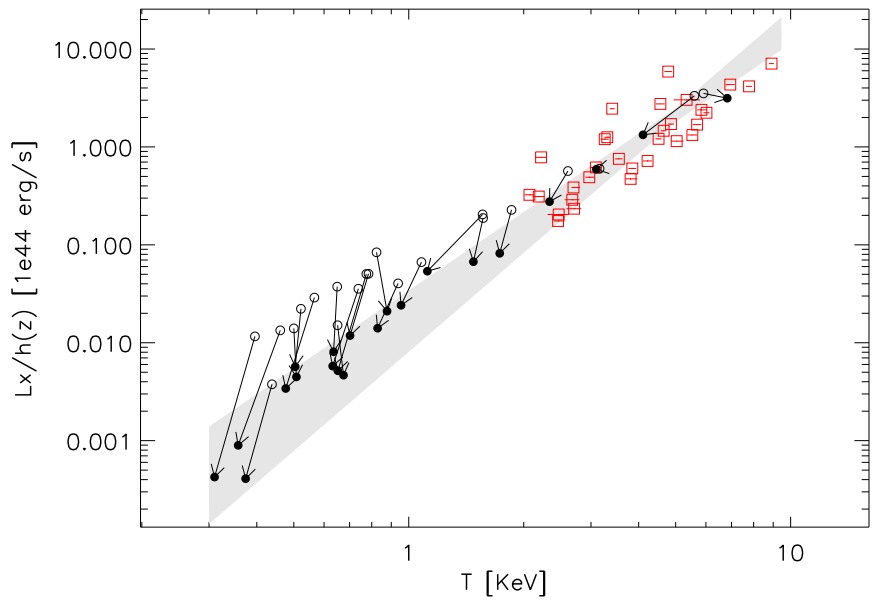

Fig. 5. X-ray luminosity as a function of the spectroscopic temperature for observations (red squares) and simulations. Arrows indicate the change of the cluster properties starting from the case without the AGN (open circles) to with the AGN (filled circles). The shaded area shows the $1 \sigma$ uncertainty of the best-fit REXCESS $L_{\mathrm{x}}-T$ scaling relation, which was derived for clusters above $2 \mathrm{keV}$.

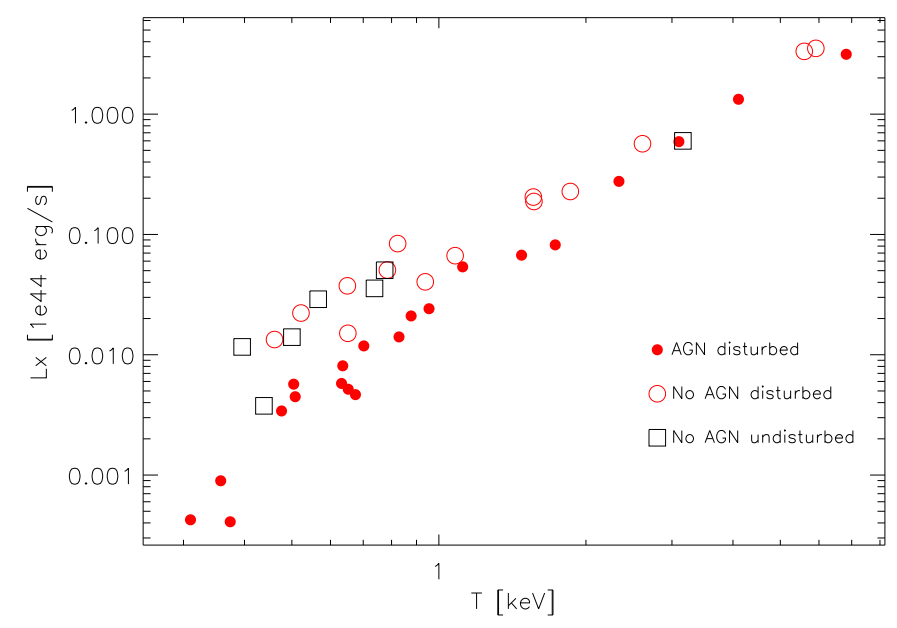

Fig. 6. X-ray luminosity as a function of the spectroscopic temperature represented by the morphological description.

would be less distorted with the same amount of feedback energy input.

This finding does not imply a complete revision of the treatment of AGN feedback in cosmological simulations for clusters. Fig. 2 of Puchwein et al. (2008) showed that there is a very good agreement between observations and simulations for the $L_{X}-T$ scaling relation. We compare here the $L_{X}-T$ relation for the simulations and for REXCESS in Fig. 5. The REXCESS data points, in red squares, were used to obtain the best-fit scaling relation in Pratt et al. (2009), which is shown in the grey region. We extend this region down to the lower temperature in the plot just for a comparison to the simulated clusters. It is clear that the simulated clusters with AGN feedback provide a much better agreement with the observed scaling relation.

Finally we examine a possible dependency of the morphological types on the physical properties of clusters in Fig. 6. The cluster morphology classification was based on the results of Fig. 4. Except for one cluster all the other six relaxed clusters are below $2 \mathrm{keV}$, and there is no other clear segregation of relaxed and unrelaxed clusters with respect to the $L_{\mathrm{X}}-T$ relation, as was found in Chon et al. (2012) and Böhringer et al. (2010).
Hence we conclude in this section with a statement that the simulated clusters have more substructures than the observations as measured by $w$ and $P_{3}$, and the degree of substructures for the simulation with the AGN feedback is much higher than the simulation without. This is the case despite the fact that the observed scaling relation, $L_{X}-T$, is better reproduced with the AGN feedback. This demonstrates that the substructure measures, $w$ and $P_{3}$, are sensitive to the included feedback physics and suggests that they should be used in addition to global properties, such as $L_{\mathrm{X}}$ and T, and radial profiles to test cluster simulations against observations.

Finally, we want to reflect on what changes in the simulated physics might ease the tension with observations. In fact some of the most recent simulations of galaxy cluster formation do get a mixture of cool-core and non-cool-core clusters (Hahn et al. 2016; Rasia et al. 2015) and might potentially also be doing better in terms of the X-ray substructure measures employed here. Both works differ from our simulations in the treatment of the hydrodynamics. Hahn et al. (2016) use the Eulerian adaptive mesh refinement code RAMSES. Rasia et al. (2015) employ a more modern SPH version with an updated interpolating kernel and artificial conduction, which allows to better resolve fluid instabilities and results in more mixing (Beck et al. 2016). While these two hydro algorithms are very different, they result in almost identical thermodynamic profiles in a non-radiative galaxy cluster simulation in which the effect of the hydrodynamics can be directly compared (Sembolini et al. 2016). These profiles also differ significantly from those obtained with classic SPH algorithms like those employed in our runs. Classic SPH codes also tend to produce somewhat more cold gas clumps due to a suppression of fluid instabilities that could otherwise shred them. This could potentially increase X-ray substructure measures. We think, however, that this is not a large effect and therefore does not play a role in the comparison of simulations with and without AGN feedback.

Our simulations compute radiative cooling based on a primordial gas composition. It would certainly be desirable to replace this with a chemical evolution and metal-dependent cooling prescription. In the synthetic X-ray analysis an ICM metallicity of 0.3 times the solar value was assumed. This is in broad agreement with typical observed values. Nevertheless, a more realistic inhomogeneous enrichment could somewhat alter the $\mathrm{X}$-ray morphology in particular in low mass objects where metal line emission often dominates over free-free Bremsstrahlung.

In terms of the AGN feedback implementation Hahn et al. (2016) and Rasia et al. (2015) inject the feedback energy in the immediate neighbourhood of the $\mathrm{BH}$ particle, while in our simulations the injection of bubbles is somewhat off-centre to mimic the observed positions of X-ray cavities/radio bubbles. AGN jets may shoot out to these radii with very limited coupling to the ICM further inside. In practice it is, however, often difficult to achieve high enough numerical resolution to resolve bubbles of realistic sizes well enough to accurately follow their dynamics. One is then left with the choice of either injecting very poorly resolved bubbles or making them somewhat larger. The adopted parameters controlling the bubble size in our simulations (see Sect. 2.1) fall in the latter regime. As the cluster-centric radius of the bubble centre is randomly chosen within 5/3 times the bubble radius, this also results in more off-centred bubbles, which could potentially disrupt an approximately spherical symmetric gas distribution more strongly and might result in larger substructure measures. In very high resolution simulations, more accurately matching the bubble properties and positions to observations is thus worth exploring. 


\section{Summary}

We used two measures of substructures, the centre shifts, $w$, and power ratios, mainly $P_{3}$, to diagnose the degree of substructures in X-ray clusters for both simulations and observations. The line-of-sight projection of a cluster introduces an ambiguity in the measured degree of substructures leading to scatter for individual clusters. Thus these measures may not be used lightly to judge an individual case, but they are very useful in treating statistical samples of clusters.

We find that a combination of $w$ and $P_{3}$ provides a reliable measure to diagnose dynamical states of clusters as found in Chon et al. (2012). This pair of parameters was used to divide the cluster samples into three classes of morphologies or equivalently dynamic states: disturbed, intermediate, and relaxed. This classification divides the REXCESS clusters into two similarly sized groups, one group of the disturbed and another of intermediate and relaxed clusters. The simulated clusters, however, are dominated by clusters with much more substructures for both types of simulations, i.e. with or without the AGN feedback. Moreover the degree of substructure is much higher for the simulated clusters with AGN feedback and all clusters with AGN feedback were found to be disturbed.

This discrepancy in the substructure analysis between observations and simulations should be considered when refining simulation models, which may be affected both by the treatment of the hydrodynamics and the sub-resolution galaxy formation physics. For example, the inclusion of AGN feedback in the simulations is necessary to reproduce the scaling relation of the observed X-ray clusters well, as was shown in Puchwein et al. (2008) and in Fig. 5. The substructure measures are, however, also sensitive to this physics. They should thus be taken into account in addition to global cluster properties and ICM profiles. In our simulations, it might, for example, be interesting to explore whether more accurate hydrodynamics in higher resolution that allows to resolve AGN bubbles better, as well as a more accurate matching of the properties of these bubbles to observations would improve the agreement with observed substructure measures.

Acknowledgements. H.B. and G.C. acknowledge support from the DFG Transregio Program TR33 and the Munich Excellence Cluster "Structure and Evolution of the Universe". G.C. acknowledges support by the DLR under grant No. 50 OR 1405. E.P. acknowledges support by the Kavli Foundation and the FP7 ERC Advanced Grant Emergence-320596.

\section{References}

Allen, S. W., Dunn, R. J. H., Fabian, A. C., Taylor, G. B., \& Reynolds, C. S. 2006, MNRAS, 372, 21

Beck, A. M., Murante, G., Arth, A., et al. 2016, MNRAS, 455, 2110

Böhringer, H., Schuecker, P., Guzzo, L., et al. 2001, A\&A, 369, 826

Böhringer, H., Matsushita, K., Churazov, E., Ikebe, Y., \& Chen, Y. 2002, A\&A, 382,804

Böhringer, H., Schuecker, P., Pratt, G. W., et al. 2007, A\&A, 469, 363

Böhringer, H., Pratt, G. W., Arnaud, M., et al. 2010, A\&A, 514, A32

Böhringer, H., Chon, G., Collins, C. A., et al. 2013, A\&A, 555, A30

Böhringer, H., Chon, G., \& Collins, C. A. 2014, A\&A, 570, A31

Böhringer, H., Chon, G., Bristow, M., \& Collins, C. A. 2015, A\&A, 574, A26

Buote, D. A., \& Tsai, J. C. 1995, ApJ, 452, 522

Chon, G., \& Böhringer, H. 2012, A\&A, 538, A35

Chon, G., Böhringer, H., \& Smith, G. P. 2012, A\&A, 548, A59

Chon, G., Böhringer, H., \& Nowak, N. 2013, MNRAS, 429, 3272

Collins, C. A., Guzzo, L., Böhringer, H., et al. 2000, MNRAS, 319, 939

Fabjan, D., Borgani, S., Tornatore, L., et al. 2010, MNRAS, 401, 1670

Hahn, O., Martizzi, D., Wu, H.-Y., et al. 2016, MNRAS, submitted [arXiv: 1509.04289]

Hasselfield, M., Hilton, M., Marriage, T. A., et al. 2013, J. Cosmol. Astropart. Phys., 7, 8

Heinz, S., Reynolds, C. S., \& Begelman, M. C. 1998, ApJ, 501, 126

Kravtsov, A. V., \& Borgani, S. 2012, ARA\&A, 50, 353

Mahdavi, A., Hoekstra, H., Babul, A., et al. 2013, ApJ, 767, 116

McCarthy, I. G., Schaye, J., Ponman, T. J., et al. 2010, MNRAS, 406, 822

McNamara, B. R., \& Nulsen, P. E. J. 2007, ARA\&A, 45, 117

McNamara, B. R., \& Nulsen, P. E. J. 2012, New J. Phys., 14, 055023

Mead, J. M. G., King, L. J., Sijacki, D., et al. 2010, MNRAS, 406, 434

Mohr, J. J., Fabricant, D. G., \& Geller, M. J. 1993, ApJ, 413, 492

Peterson, J. R., Paerels, F. B. S., Kaastra, J. S., et al. 2001, A\&A, 365, L104

Planck Collaboration XIII. 2016, A\&A, in press, DOI: $10.1051 / 0004-6361 / 201525830$

Planck Collaboration XXIV. 2016, A\&A, in press, DOI: $10.1051 / 0004-6361 / 201525833$

Planelles, S., Borgani, S., Fabjan, D., et al. 2014, MNRAS, 438, 195

Poole, G. B., Fardal, M. A., Babul, A., et al. 2006, MNRAS, 373, 881

Pratt, G. W., Croston, J. H., Arnaud, M., \& Böhringer, H. 2009, A\&A, 498, 361

Puchwein, E., Sijacki, D., \& Springel, V. 2008, ApJ, 687, L53

Puchwein, E., Springel, V., Sijacki, D., \& Dolag, K. 2010, MNRAS, 406, 936

Rasia, E., Meneghetti, M., \& Ettori, S. 2013, Astron. Rev., 8, 40

Rasia, E., Borgani, S., Murante, G., et al. 2015, ApJ, 813, L17

Sembolini, F., Yepes, G., Pearce, F. R., et al. 2016, MNRAS, 457, 4063

Sijacki, D., \& Springel, V. 2006, MNRAS, 366, 397

Sijacki, D., Springel, V., Di Matteo, T., \& Hernquist, L. 2007, MNRAS, 380, 877

Springel, V., \& Hernquist, L. 2002, MNRAS, 333, 649

Springel, V., Di Matteo, T., \& Hernquist, L. 2005a, MNRAS, 361, 776

Springel, V., White, S. D. M., Jenkins, A., et al. 2005b, Nature, 435, 629

Vikhlinin, A., Kravtsov, A. V., Burenin, R. A., et al. 2009, ApJ, 692, 1060 